\title{
Influence length of single-beam propagation in doped glass and polymer optical fibres
}

\author{
Maryam Karimi ${ }^{1}$ and Faramarz E. Seraji ${ }^{2}$ \\ ${ }^{1}$ Physics Group, Razi University, Kermanshah, Iran \\ ${ }^{2}$ Optical Communication Group, Iran Telecom Research Center, 14399 Tehran, \\ Iran, E-mail: feseraji@itrc.ac.ir
}

Received: 14.01.2010

\begin{abstract}
For characterisation of doped optical fibres (DOFs) based on single-beam propagation, one requires a maximum DOF length along which a signal power can propagate. In this work we have solved the rate equation for the single-beam propagation in both lossy and lossless DOFs, introduced an influence length as a maximum propagation length and then determined it analytically. With the analytical results obtained, the main regularities for the influence length have been simulated for the glass optical fibres doped with $\mathrm{Ge} / \mathrm{Al} / \mathrm{Er}$ operating at arbitrary wavelengths under lossless and lossy conditions. Also, the influence length has been determined for some kinds of co-dopants present in both polymer and glass optical fibres. The influence length thus defined may be useful in any measurements based on the single-beam propagation in DOFs.
\end{abstract}

Keywords: influence length, doped optical fibres, single-beam propagation, fibre lasers and amplifiers

PACS: 42.81._i, 42.25.Bs; 42.79._e; 42.65.Yj, 42.55.Wd

UDC: 535.3

\section{Introduction}

Optical fibre lasers and amplifiers made as doped optical fibres (DOFs) have many practical applications in industries and medicine. A number of characteristics of DOFs should be determined prior to designing these components. Some parameters of DOFs, such as dopants' concentration and fluorescence, steady-state lifetime, absorption and emission cross-sections, attenuation coefficient, core radius, and refractive index, are the main factors which affect fibre lasers and amplifiers [1]. Some other parameters (gain, saturated gain, noise figure, optimum fibre length, etc.) depend on the above-said factors [2]. Moreover, there are some additional factors, e.g., availability of co-dopants and their concentrations, conditions of alignment of the dopants in the crystalline form of host materials, which influence those main parameters, too [2]. The parameters describing any manufactured DOF are often unique [3].

For characterisation of DOFs using double [4] and triple cut-back methods [5, 6], or a similar technique suggested in [7], single-beams are usually utilised in the experiments. In another experimental technique [8], a single-beam as a test pulse has also been used for simultaneous measurements of nonlinearity and group-velocity dispersion. Thus, for a 
proper choice of length of a DOF under test we should know the penetration depth of single-beam propagation along the DOF, in order to detect the output.

In this paper, we will introduce an influence length (IL) as a penetration depth of single-beam and a length of DOF for which the power at its output end drops down as small as $1 / e^{2}$ of the input power. This parameter depends on the main DOF parameters. We will compare the IL of glass and polymer DOFs showing a background loss and lacking it. Then an important conclusion follows that the optimum lengths of DOFs are best to be shorter than the IL.

\section{IL for single-beam propagation in DOFs}

Using of the rate equation is the simplest way to verify parameters of fibre amplifiers and lasers [2]. Our model is based on a three-level system, with a fast decay of third level operated only by a single beam, with any wavelength equal to operating wavelength of a lossy DOF under test. In high-loss DOFs, like dye-doped polymer optical fibres or doped glass ones, the background loss term is inserted into the rate equations basing on phenomenological approach [9-11]. Therefore, under single-beam operation of the DOF, the rate equations for the input intensity and densities are as follows [9-11]:

$$
\begin{aligned}
& \frac{\partial I}{\partial z}=-2 \pi\left(\sigma_{e} N_{2}-\sigma_{a} N_{1}\right) I-\alpha I, \\
& \frac{\partial N_{2}}{\partial t}=-\frac{N_{2}}{\tau}+\left(\sigma_{a} N_{1}-\sigma_{e} N_{2}\right) \frac{I}{h v}, \\
& N_{1}+N_{2}=N_{t},
\end{aligned}
$$

where $I$ is the input intensity, $N_{1}, N_{2}$ and $N_{t}$ respectively the ground, metastable and the total charge densities, $\sigma_{a}$ and $\sigma_{e}$ respectively the absorption and emission crosssections, $\tau$ the steady-state lifetime of a dopant on its metastable level, $h$ the Planck constant, $\alpha$ the loss coefficient of the DOF and, finally, $v$ the input-power frequency. The effect of amplified spontaneous emission may be disregarded when the input power of the DOF under test is low and/or its length is short enough [12-15]. Using Eqs. (2) and (3), we obtain

$$
\frac{\partial N_{2}}{\partial t}=-N_{2}\left\{\frac{1}{\tau}+\left[\left(\sigma_{e}+\sigma_{a}\right) / h v\right] I\right\}+\left(\frac{\sigma_{a} N_{t}}{h v}\right) I .
$$

In the given DOF, the input intensity is assumed as a slow-varying function of time, so that Eq. (4) represents a linear first-order differential equation with $N_{2}$ being timevariant. The solution reads as follows:

$$
N_{2}=\frac{\sigma_{a} N_{t} I}{h v+\left(\sigma_{e}+\sigma_{a}\right) I}\left\{1-\exp \left[-\left(\frac{1}{\tau}+\frac{\left(\sigma_{e}+\sigma_{a}\right)}{h v} I\right) t\right]\right\}+C^{\prime},
$$

where the $C^{\prime}$ constant can be determined from the boundary condition $t \rightarrow 0 \Rightarrow N_{2} \rightarrow 0$.

To achieve a steady-state condition, the exponential term in Eq. (5) should equal to -1 . Therefore, we introduce the steady-state time $T$ as follows: 


$$
T=\frac{1}{(1 / \tau)+I\left(\sigma_{e}+\sigma_{a}\right) / h v},
$$

Here the steady-state time is of the order of metastable lifetime $\tau$ for DOFs, i.e., of the order of millisecond for the case of Er-doped fibre amplifiers and nanosecond for organic-doped polimer optical fibres [10]. Due to high input powers, the $T$ value in the input region of erbium-doped fibre amplifiers rapidly reaches the steady state. When the pump and signal simultaneously enter the Er-doped fibre amplifier, Eq. (6) should be changed to [4]

$$
T=\frac{1}{(1 / \tau)+\left(\sigma_{a p} / h v_{p}\right) I_{p}+\left[\left(\sigma_{e s}+\sigma_{a s}\right) / h \nu_{s}\right] I_{s}} .
$$

Eq. (7) testifies that, under simultaneous presence of the pump and signal fields in the DOF, the amplifier achieves its steady state quicker than in the case of only one of the fields present. The pump power decreases along the DOF length, while the signal power then increases and reaches its maximum at the output end $[2,16]$. This makes the $T$ value to be nearly constant.

Basing on Eq. (5) for the steady-state of dopant atoms, one can assume that the electron density of the second level is constant. The density $N_{2}$ of the second level varies as

$$
N_{2}=\frac{\sigma_{a} N_{t} I}{h v+\left(\sigma_{e}+\sigma_{a}\right) I}
$$

Supposing the steady-state condition and substituting $N_{2}$ from Eq. (8) and $N_{1}$ from Eq. (3) into Eq. (1), we obtain an equation describing the input intensity variation versus the length of a lossy medium:

$$
\frac{\partial I}{\partial z}=2 \pi\left[-\frac{\sigma_{a} N_{t}(h v / \tau)}{(h v / \tau)+\left(\sigma_{e}+\sigma_{a}\right) I}\right] I-\alpha I .
$$

By integrating Eq. (9) we obtain

$$
\int\left(\frac{\left[h \gamma / \tau+\left(\sigma_{e}+\sigma_{a}\right) I\right]}{2 \pi \sigma_{a} N_{t}(h \gamma / \tau)+\alpha\left[h \gamma / \tau+\left(\sigma_{e}+\sigma_{a}\right) I\right] I} d I\right)=-z-C_{\text {lossy }},
$$

where $C_{\text {lossy }}$ means the constant of integration obtained with the boundary condition $\left(I=I_{0} \Rightarrow z=0\right)$. It is seen that $C_{\text {lossy }}$ depends upon the $I_{0}$ parameter and the type of DOF.

The model involving Eq. (10) is similar to that used for determination of the background loss in Er-doped fibre amplifiers [5]. This integral can simply be solved using a common table of integrals [17], provided that the absorption and emission crosssections are known and the loss is determined on this basis. However, these parameters are unknown within the model suggested by us and so we have to find a set of four functions in order to determine the given parameters. Because of the presence of arctangent function in the set of functions derived above, the analytical solution of our 
model would be rather complex. Desiring to solve the model analytically, we convert the solution integral into a logarithmic form, by using the following definitions:

$$
a=2 \pi \sigma_{a} N_{t}\left(\frac{h v}{\tau}\right), U=\frac{h v}{\tau}+\left(\sigma_{e}+\sigma_{a}\right) I, \gamma=\frac{h v}{\tau} .
$$

Then the 1. h. s. of Eq. (10) changes to

$$
\int \frac{U d U}{(a-\alpha U)(U-\gamma)}=\left[\int \frac{d U}{(a-\alpha U)}+\gamma\left(\int \frac{A \cdot d U}{(a-\alpha U)}+\int \frac{B \cdot d U}{(U-\gamma)}\right)\right],
$$

with $A$ and $B$ being defined as

$$
\begin{aligned}
& A=\frac{1}{U-\gamma}, \text { if } U=\frac{a}{\alpha} \Rightarrow A=\frac{1}{(a / \alpha-\gamma)}, \\
& B=\frac{1}{(a-\alpha U)}, \text { if } U=\gamma \Rightarrow B=\frac{1}{\alpha(a / \alpha-\gamma)} .
\end{aligned}
$$

Changing the above variables and solving the integral Eq. (11), we arrive at the solution sought after:

$$
(\alpha U+a)^{(a / \alpha)}(U-v)^{v}=\exp \left[-\alpha\left(z+C_{\text {lossy }}\right)(a / \alpha+v)\right] .
$$

Eq. (13) gives a variation of the input signal intensity with respect to the length in the lossy DOF. If the background loss in the DOF is negligible, the solution of the coupled rate equations (i.e., Eqs. (1), (2) and (3)) reads as [4, 16]

$$
\frac{1}{2 \pi \sigma_{a} N_{t}} \ln I+\frac{\sigma_{e}+\sigma_{a}}{2 \pi \sigma_{a} N_{t}(h \gamma / \tau)} I=-\left(z+C_{\text {lossless }}\right) \text {. }
$$

Again, the integration constants are determined from the boundary condition ( $I=I_{0} \Rightarrow z=0$ ). As a result, from Eq. (13) we get for the lossy medium

$$
C_{\text {lossy }}=\frac{\ln \left[\left(\alpha U_{0}-a\right)^{-(a / \alpha)} \cdot\left(U_{0}-v\right)^{v}\right]}{(a / \alpha-v)},
$$

where $U_{0}=h v / \tau+\left(\sigma_{e}+\sigma_{a}\right) I_{0}$ depends on the input power in that medium. For the lossless medium Eq. (14) yields in

$$
C_{\text {lossless }}=-\left[\frac{1}{2 \pi \sigma_{a} N_{t}} \ln I_{0}+\frac{\sigma_{e}+\sigma_{a}}{2 \pi \sigma_{a} N_{t}(h v / \tau)} I_{0}\right] .
$$

Hence, using Eqs. (13) and (15) or, alternatively, Eqs. (14) and (16) enables one to find the equations governing the power variations along the DOF length for the lossy and lossless conditions, respectively. When the intensity in the DOF becomes $1 / e^{2}$ of the primary input intensity, the IL for the single-beam propagation in DOFs may be determined as

$$
\begin{aligned}
Z_{\inf (\text { lossy })} & =-\frac{a}{\alpha}\left[\ln \alpha+\ln \left(\frac{h v / \tau+\left(\sigma_{e}+\sigma_{a}\right) I_{0} / e^{2}-(a / \alpha)}{h v / \tau+\left(\sigma_{e}+\sigma_{a}\right) I_{0}-(a / \alpha)}\right)\right], \\
& +\gamma \ln \frac{h v / \tau+\left(\sigma_{e}+\sigma_{a}\right) I_{0} / e^{2}-\gamma}{h v / \tau+\left(\sigma_{e}+\sigma_{a}\right) I_{0}-\gamma}
\end{aligned}
$$




$$
Z_{\text {inf }(\text { lossless })}=1 /\left(\pi \sigma_{a} N_{t}\right)+0.8647\left[\frac{\sigma_{a}+\sigma_{e}}{2 \pi \sigma_{a} N_{t}(h v)}\right] I_{0},
$$

where Eq. (17) and Eq. (18) refer for the lossy and lossless media, respectively.

\section{Simulation results}

We have used a bisectional method for solving Eqs. (13) and (14) [15]. Fig. 1a and Fig. $1 b$ show variations of the output power with the DOF length in Ge/Al/Er DOF (EDOF) for a number of wavelengths and values of the other parameters. Here the core diameter $4.3 \mu \mathrm{m}$ and the steady-state lifetime $10 \mathrm{~ms}$ [2] are taken for the EDOF. When the background loss in the EDOF remains negligible, the variation of the power with respect to the DOF length is semi-linear, irrespective of the wavelength (see Fig. 1a and Eq. (14)). If we assume a background loss for the EDOF, the dependence of the power variation on the length is semi-exponential (see Fig. 1b and Eq. (13)) and the maximum length of beam influence is shorter than in the previous case.
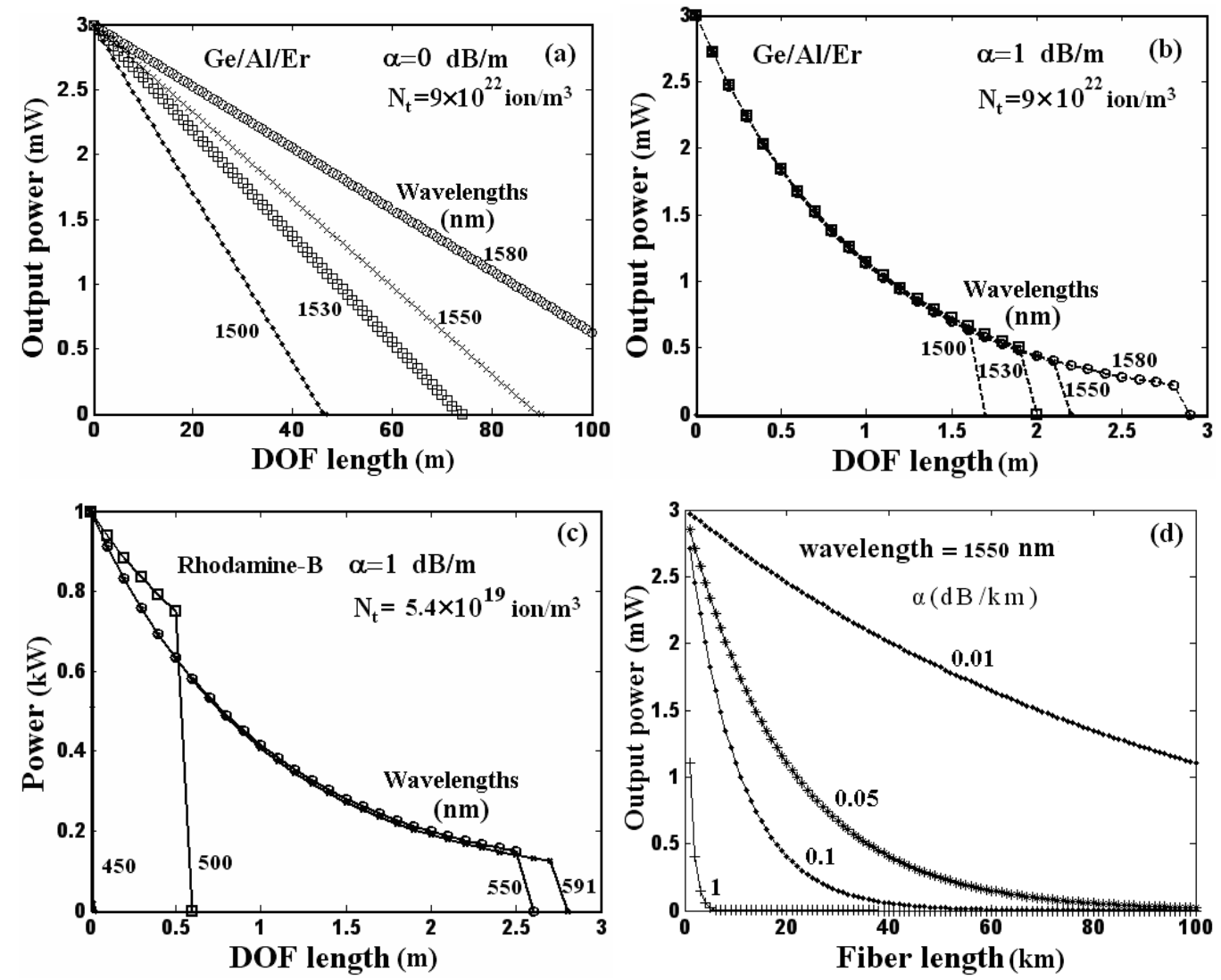

Fig. 1. Variations of output power vs. DOF length for the cases of (a) lossless erbium-doped fibre, (b) lossy erbium-doped fibre, (c) lossy RB-DPOF, and (d) single-mode fibre.

In Fig. 1c the same dependence is plotted for a polymer optical fibre doped with Rhodamine-B (RB-DPOF) and different wavelengths. Here the following parameters are 
used: $\alpha=1 \mathrm{~dB} / \mathrm{m}, N_{t}=5.4 \times 10^{19} \mathrm{ion} / \mathrm{m}^{3}$, the core diameter of $500 \mu \mathrm{m}$, and $\tau=2.85 \mathrm{~ns}$ $[9,10]$. It is obvious that the length variation of the power for the lossy RB-DPOF is semi-linear, too. When comparing the lossy EDOF with the lossless RB-DPOF, one can clearly observe a wavelength effect in the lossless medium. At the same time, different wavelengths have almost the same effect for the lossy medium or, at least, it is hardly distinguishable. The exponential power variation for the single-mode fibre with $\alpha=1 \mathrm{~dB} / \mathrm{km}$ is displayed in Fig. 1d. Comparing Fig. 1d with Fig. $1 \mathrm{~b}$ and Fig. 1c, we conclude that the lossy DOFs reveal the behaviour close to that characteristic of singlemode ones.

Basing on Eqs. (17) and (18), we have plotted the IL for the lossless and lossy glass EDOFs at different wavelengths (see Fig. 2a and Fig. 3a, respectively). The corresponding 3D diagrams are depicted in Fig. 2b and Fig. $3 b$.

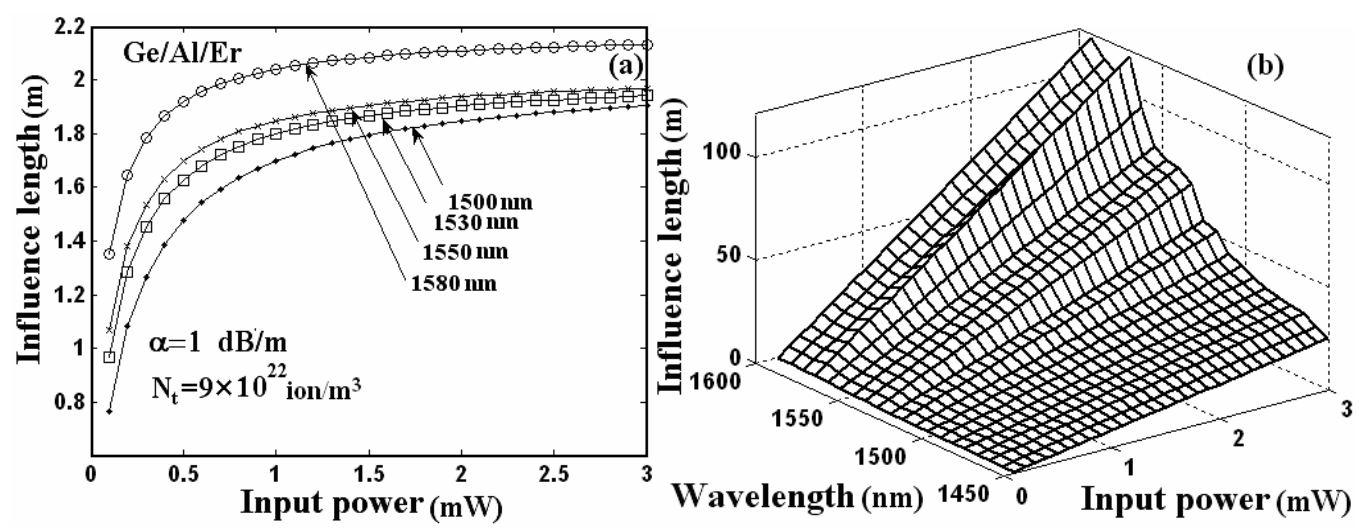

Fig. 2. Dependences of IL on the input power at different wavelengths for the lossless EDOF.
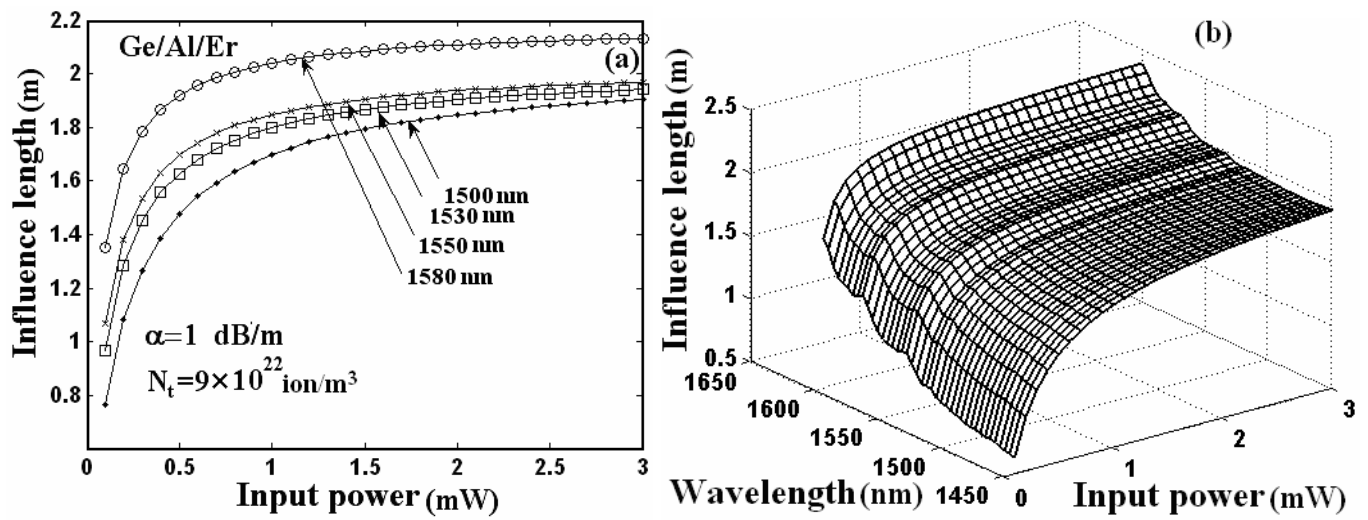

Fig. 3. Dependences of IL on the input power at different wavelengths for the lossy EDOF.

As seen from Fig. 2 and Fig. 3, the IL varies linearly with the input power and the wavelength when the background loss of the EDOF is absent. The presence of losses in 
the EDOF makes the trend change slowly with the input power. In other words, the background loss induces the power saturation in the EDOF. The ILs at longer wavelengths are larger, when compare with shorter ones.
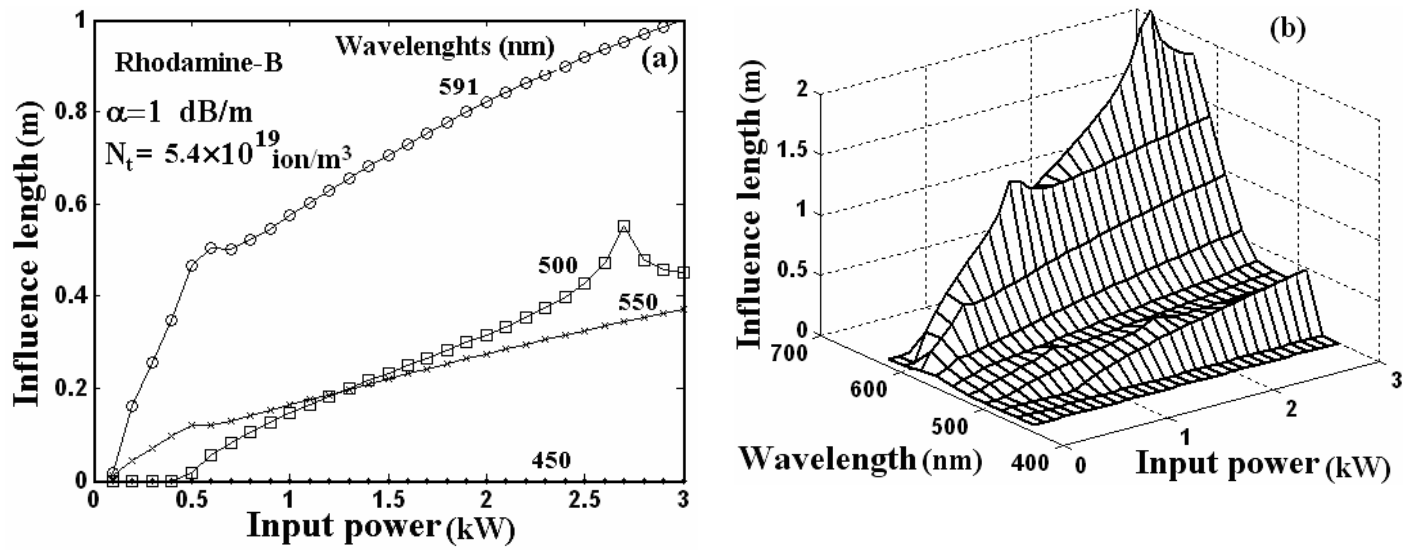

Wavelength (nm) $\quad 400 \quad 0 \quad \operatorname{Input} \operatorname{power}(\mathrm{kW})$

Fig. 4. Dependences of IL on the input power at different wavelengths for the RB-DPOF.
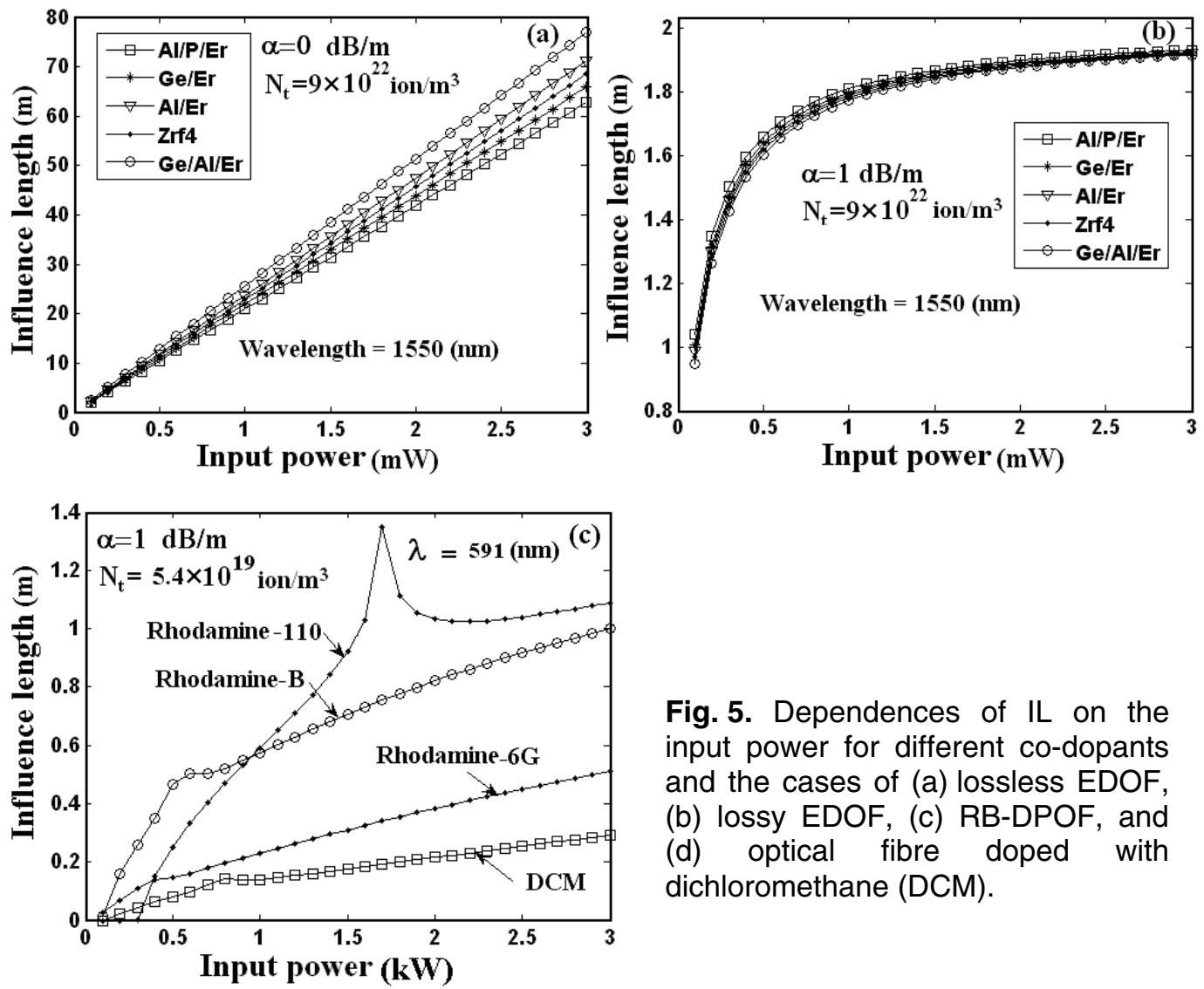

Fig. 5. Dependences of IL on the input power for different co-dopants and the cases of (a) lossless EDOF, (b) lossy EDOF, (c) RB-DPOF, and (d) optical fibre doped with dichloromethane (DCM).

Fig. 4a shows the dependences of IL of the RB-DPOF on the wavelength, while the relevant 3D diagrams are depicted in Fig. 4b. Here the IL behaves nonlinearly with increasing input power. At some wavelengths, the IL has a peak at a certain input power and after the peak point the IL value changes slowly. In other terms, the IL reveals little variation with increasing input power when there are high losses in the DOF, irrespective 
of whether we deal with the polymer or glass DOFs. In all the cases, the presence of losses imposes the IL to become shorter than that for the lossless DOFs.

Fig. 5 summarises the effect of co-dopants in the glass and polymer DOFs for the lossless and lossy conditions (the densities being $N_{t}=9 \times 10^{22} \mathrm{ion} / \mathrm{m}^{3}$ and $N_{t}=5.4 \times 10^{19} \mathrm{ion} / \mathrm{m}^{3}$ ). It is interesting to note that the ILs under the both conditions mentioned above maintain their major features for different co-dopants. In the lossy case, the differences among the ILs due to the co-dopants are weak, irrespective of the input power (see Fig. 5b). In what concerns the optical fibres doped with Rhodamine-B, Rhodamine-6G, Rhodomain-110 and Dichloromathane dyes, the slopes of the IL variations decrease and there appears a sharp peak at the input power of about $1.68 \mathrm{~kW}$ for Rhodomain-110.

\section{Conclusion}

We have derived the relations for single-beam propagation for the both lossy and lossless DOFs by solving analytically the rate equations. Using these relations, we have analysed the optical power propagation in the DOFs based on silica and polymers. Issuing from that analysis, an IL for the single-beam propagation along the glass and polymer DOFs may be introduced. Our results show that the IL variations slow down under the lossy conditions. The dependence of the IL on the input power is almost linear when the background loss in the EDOF is negligible and becomes nonlinear otherwise. The IL turns out to be nearly a constant with respect to the input power in the case of EDOFs. The IL in the RB-DPOF has a peak at some wavelengths. The effect of co-dopants in the lossless conditions is more pronounced when compare with the lossy DOFs.

Finally, the results of our analysis demonstrate that the IL for the single-beam propagation in DOFs is always longer than that for the case of double-beam propagation.

\section{References}

1. Guokui Liu and Bernard Jacquier, Eds. Spectroscopic properties of rare earths in optical materials. New York, LLC: Springer-Verlag (2005).

2. Desurvire E, Erbium doped fiber amplifiers: principles and applications. New York: Wiley (1994).

3. Barnes W L, Laming R I, Tarbox E J and Morkel P R, 1991. Absorption and emission cross section of $\mathrm{Er}^{3+}$ doped silica fibers, IEEE J. Quant. Electron. 27: 1004-1010.

4. Karimi $M$ and Seraji F E, Experimental technique to determine absorption and emission cross Sections of erbium doped fiber optics. Proc. Iranian Phys. Conf. (2008), p. 62.

5. Karimi $M$ and Seraji $F$ E, A Novel model to determine fluorescence and loss coefficient in doped optical fiber used in WDM transmission dystems. Proc. Asia Opt. Fiber Commun. Optoelectron. Expo Conf. ( 2008), SuK7.

6. Karimi M and Seraji F E, A Proposed method to simultaneously measure doped optical fibers parameters, Proc. Iran. Phys. Conf., p.17. 
7. Karimi M and Seraji F E, 2010. Experimental technique for simultaneous measurement of absorption-, emission cross-section, and background loss coefficient in doped optical fibers. Appl. Phys. B. 98: 113-117.

8. Nguyen T N, Chartier T, Thual M, Besnard P, Provino L, Monteville A and Traynor N, 2007. Simultaneous measurement of anomalous group-velocity dispersion and nonlinear coefficient in optical fibers using soliton-effect compression. Opt. Commun. 278: 60-65.

9. Kobayashi T, Kuiki K, Imai N, Tamura T, Sasaki K and Koike Y, 1999. High power polymer optical fiber lasers and amplifiers. Proc. SPIE Conf. Organic Photon Mat. and Dev. 3623: 206.

10. Karimi M, Granpayeh N and Moravvej Farshi M K, 2004. Analysis and design of a dye-doped polymer optical fiber amplifier. Appl. Phys. B. 78: 387-396.

11. Desurvire E and Simpson J, 1991. Analysis of distributed erbium-doped fiber amplifiers with fiber background loss. IEEE Photon. Technol. Lett. 3: 625-628.

12. Adikan F R M, Noor A S M and Mahdi M A, 2004. Optimum pumping configuration for L-band EDFA incorporating ASE pump source. IEEE Photon. Technol. Lett. 16: 1465-1467.

13. Desurvire E, Zirngibl M, Presby H M and DiGiovanni D, 1991. Characterization and modeling of amplified spontaneous emission in unsaturated erbium doped fiber amplifiers. IEEE Photon. Technol. Lett. 3: 127-129.

14. Desurvire E and Simpson J, 1989. Amplification of spontaneous emission in erbiumdoped single-mode fibers. IEEE J. Lightwave Technol. 7: 835-845.

15. Becker PC, Olsson NA and Simpson JR. Erbium doped fiber amplifiers: fundamentals and technology. London: Academic Press (1999).

16. Karimi M and Seraji Faramarz E, Mono-beam propagation in low and high loss Erdoped optical fiber. Proc. 2nd Int. Conf. on Elect. Eng. Design and Technol. ICEEDT'08, (2008).

17. Polyanin A D and Manzhirov A V. Handbook of mathematics for engineers and scientists. Boca Raton-London: Chapman \& Hall/CRC Press (2007).

Maryam Karimi and Faramarz E. Seraji, 2010. Influence length of single-beam propagation in doped glass and polymer optical fibres. Ukr.J.Phys.Opt. 11: 90-98.

Анотація. Умовою охарактеризування легованих оптичних однопроменевих волокон (ЛОВ) $\epsilon$ забезпечення їх максимальної довжини вздовж якої поширюється сигнал. В даній роботі ми розв'язали рівняння для поширення променя у ЛОВ з втратами та без них, ввівши ефективну довжину, як максимальну довжину поширення, яка потім визначалась аналітично. На основі аналітично отриманих результатів були промодельовані основні закономірності ефективної довжини для скляних оптичних волокон, легованих Ge/Al/Er, які функиіонували на різних довжинах хвиль, за умови наявності і відсутності втрат. Крім иъього, ефективна довжина була визначена для полімерних і скляних волокон з певним типом домішок. Таким чином, продемонстровано, щяо ефективна довжина може бути корисною при однопроменевих вимірюваннях у ЛОВ. 